\title{
Early intervention to improve hand function in hemiplegic cerebral palsy
}

\author{
Anna Purna Basu ${ }^{1,2}$ *, Janice Pearse ${ }^{1,3}$, Susan Kelly ${ }^{3}$, Vicki Wisher ${ }^{3}$ and Jill Kisler ${ }^{1,2}$ \\ 1 Institute of Neuroscience, Newcastle University, Newcastle upon Tyne, UK \\ 2 Department of Paediatric Neurology, Newcastle upon Tyne Hospitals NHS Foundation Trust, Newcastle upon Tyne, UK \\ ${ }^{3}$ Paediatric Physical Therapy, Newcastle upon Tyne Hospitals NHS Foundation Trust, Newcastle upon Tyne, UK
}

Edited by:

Gavin John Clowry, Newcastle

University, UK

\section{Reviewed by:}

Jennifer Christy, University of

Alabama at Birmingham, USA

Andrea Guzzetta, University of Pisa,

Italy

\section{*Correspondence:}

Anna Purna Basu, Institute of Neuroscience, Level 3, Sir James Spence Institute, Newcastle University, Royal Victoria Infirmary,

Queen Victoria Road, Newcastle upon Tyne NE1 4LP, UK

e-mail:anna.basu@ncl.ac.uk
Children with hemiplegic cerebral palsy often have marked hand involvement with excessive thumb adduction and flexion and limited active wrist extension from infancy. Postlesional aberrant plasticity can lead to progressive abnormalities of the developing motor system. Disturbances of somatosensory and visual function and developmental disregard contribute to difficulties with hand use. Progressive soft tissue and bony changes may occur, leading to contractures, which further limit function in a vicious cycle. Early intervention might help to break this cycle, however, the precise nature and appropriateness of the intervention must be carefully considered. Traditional approaches to the hemiplegic upper limb include medications and botulinum toxin injections to manage abnormalities of tone, and surgical interventions. Therapist input, including provision of orthoses, remains a mainstay although many therapies have not been well evaluated. There has been a recent increase in interventions for the hemiplegic upper limb, mostly aimed outside the period of infancy. These include trials of constraint-induced movement therapy (CIMT) and bimanual therapy as well as the use of virtual reality and robot-assisted therapy. In future, non-invasive brain stimulation may be combined with therapy. Interventions under investigation in the infant age group include modified CIMT and action observation therapy. A further approach which may be suited to the infant with thumb-in-palm deformity, but which requires evaluation, is the use of elastic taping. Enhanced cutaneous feedback through mechanical stimulation to the skin provided by the tape during movement has been postulated to modulate ongoing muscle activity. If effective, this would represent a low-cost, safe, widely applicable early intervention.

Keywords: cerebral palsy, early intervention, upper limb, elastic taping, thumb-in-palm deformity, hemiplegia, therapy, orthoses

\section{INTRODUCTION}

Cerebral palsy (CP) is the commonest cause of neurological disability in children. The upper limbs are often affected, with significant wrist and hand involvement from an early age (1). Persisting from infancy, affected children may have abnormal hand postures such as thumb adduction and/or flexion with limited wrist extension, as well as more proximal abnormalities of upper limb tone, posture, and function, which also impact on hand use. The particular focus of this article is on the factors influencing hand structure and function in hemiplegic cerebral palsy (HCP), and the options for early intervention.

\section{INTER-RELATIONSHIP BETWEEN BODY STRUCTURE AND FUNCTION, ACTIVITIES AND PARTICIPATION}

The international classification of functioning, disability and health (ICF) (2) provides a framework, which describes the interrelationship between body structure and function, activities and participation, as well as the influence of environmental and personal factors. In the case of hand function in HCP, we see how closely each factor impacts on the others, as discussed below. This can lead to a vicious cycle of deteriorating structure and function and maladaptive activity-dependent plasticity, but can also offer hope for early intervention approaches to break the cycle.

\section{CENTRAL NERVOUS SYSTEM DEFICITS IN HCP}

Hemiplegic cerebral palsy affects around 1 in 1300 live births (3) and has a range of etiologies including neuronal migration abnormalities, periventricular leukomalacia, intracranial hemorrhage, and infarction. The common feature is disturbed cerebral control of motor function. A large component relates to corticospinal tract damage, as the corticospinal tract is the major descending tract controlling skilled, fractionated, voluntary hand movements (4). In addition, there is often extrapyramidal involvement (5); some patients have marked dystonia. As well as disruption of motor pathways, there are often sensory impairments including astereognosis (6-9), which impact detrimentally on hand function. These sensory impairments may reflect additional damage to ascending thalamocortical pathways $(10,11)$ and/or somatosensory cortical areas (9), as well as failure of sensorimotor integration (12). Disturbances of visual function, including but not limited to hemianopia, further contribute to difficulties with hand use (13). Deficits in motor planning and motor imagery as well 
as broader deficits in executive function are also seen (14-16). Finally, a minority of patients have significant learning disability, which can also impact adversely on the development of hand function $(17,18)$.

\section{ROLE OF ACTIVITY-DEPENDENT PLASTICITY WITHIN THE CENTRAL NERVOUS SYSTEM}

In addition to structural damage from the initial insult, progressive maladaptive changes occur within the central nervous system due to activity-dependent plasticity. This has been particularly studied within the corticospinal tract (19). Neurophysiological studies in humans indicate that in healthy term infants, corticospinal fibers from each hemisphere project to each side of the spinal cord (bilateral system), with gradual progression to a predominantly crossed projection within the first 2 years of life (20). If this system is perturbed by unilateral perinatal stroke, remaining corticospinal projections from the affected hemisphere may be gradually displaced over time by uncrossed projections from the undamaged hemisphere. This is in general associated with a worse functional outcome than the normal pattern of predominantly crossed corticospinal projections from each hemisphere (21). However, the relationship between corticospinal tract reorganization and functional outcome is complicated, and the nature, timing, and size of the lesion also play a role (22). A marked degree of corticospinal tract reorganization can occur (23).

Progressive maladaptive central nervous system processes in HCP are not limited to the corticospinal tract. Development of spinal cord segmental circuitry is influenced by corticospinal tract activity. If this is disrupted experimentally through transient motor cortex inhibition during early development, subsequent patterns of spinal cord circuitry including interneuron development are abnormal and immature (24-26). In addition, the M1 motor cortical map does not develop normally (27). However, processes such as early restraint of the unaffected limb combined with motor training (28), can reverse to an extent the changes in spinal cord circuitry, corticospinal tract projections, and M1 motor map abnormalities as well as improve motor outcome. Considering this in light of the ICF framework demonstrates that it is possible to alter structure and function either positively or negatively through modulation of activity.

\section{EFFECTS ON BODY STRUCTURE AND FUNCTION}

The implications of the above central nervous system changes on upper limb tone, posture, reflexes, and function in congenital hemiplegia are well-known but take time to emerge (17). In the first months of life the typical signs of a hemiplegia are not yet present, although qualitative abnormalities of movement may be detected (29). In addition, many healthy neonates and young infants will demonstrate features such as a thumb-in-palm posture, which disappear over the first few months of life (30). In contrast, the infant developing HCP will often have a closed hand posture and flexed and adducted thumb persisting beyond the first few months.

The development of asymmetrical hand function shows significant variation in infants with HCP; however, pronation of the forearm and thumb abnormalities is most frequently seen (31). As the infant develops reaching and grasping abilities on the unaffected side, parents start to notice a strong hand preference, with lack of use of the affected hand. The fingers are flexed, and the thumb is often adducted and flexed, resulting in the "thumb-in-palm" deformity (32). Dislocation may occur at the metacarpophalangeal joint, with hypermobility at the proximal interphalangeal joints causing swan-neck deformity, which impacts negatively on opposition and grasp (33). The thumb adduction also impacts on grasp, especially pincer and whole hand grasp (34). In a study of children age $4-14$ years with CP, marked structural deformities affecting hand function were noted even in the youngest children, without significant age-related increases (1). Another study also reported structural deformities even at age 2 years (31).

More proximal upper limb deficits in hemiplegia also affect hand use. Increased muscle tone is noted predominantly in the upper limb flexor muscles with extensor weakness. Dynamically increased muscle tone is particularly clear in biceps brachii during physical activity such as walking, running, and even moving the dominant hand. Pectoralis major, the forearm flexors, and pronator teres exhibit hypertonia in some children, pulling the shoulder into flexion, adduction, and internal rotation, the forearm into pronation, and the wrist into flexion, often with ulnar deviation. Active forearm supination and wrist extension are limited (32). While some of the increased tone is due to spasticity or rigidity, there are also secondary biomechanical changes in the muscles leading to hypertonus, which is independent of recorded EMG activity, but which is temporarily reduced through muscle stretching ("plasticity") $(35,36)$. Over time such increased tone can lead to the development of contractures. In addition, there is reduced limb growth on the affected side. Difficulty with selective muscle control and/or excessive co-contraction can discourage use of the hemiplegic limb which in turn compounds muscle weakness (37). Effective bimanual function is often limited (38), with increased reliance on one-handed strategies (39).

\section{EFFECT ON ACTIVITY AND PARTICIPATION}

Many daily activities require the hands to perform different movements at the same time in a coordinated way. Generally, the nondominant hand assists by holding and stabilizing objects while the dominant hand performs more precise functional movements. The child with HCP has to battle with a number of hindrances in order to use the affected hand, and may make an active choice to use the "less-affected" hand alone. [Note that subtle deficits in function are present on the side contralateral to the hemiplegia - hence the term "less-affected hand" $(40,41)]$. However, particularly for young children, the reasons for neglecting to use the affected hand are not always clear and the concept of "developmental disregard" has been coined (42). This has been described as the discrepancy between capacity and performance with the affected hand (43). Recent EEG studies indicate an increased cognitive load for movement preparation with the affected hand during a bimanual task (43). This could contribute to a preference for unimanual task performance. Compensatory strategies commonly observed include use of the teeth or stabilizing objects between the knees or against the body instead of using the affected hand. While children may be able to perform some "bimanual" tasks largely one-handed using such strategies, task completion 
time often remains prolonged (44). This can lead children to seek the assistance of others, or to avoid certain activities (45). Parents, teachers, and friends may step in too early to provide assistance. Even where the use of both hands is actively encouraged, very young children may not understand why this is important. However, as children get older they begin to recognize things they would like to be able to do more easily, particularly during the teenage years when personal independence becomes increasingly important. Personal and familial emotions and attitudes regarding the hemiplegia will influence for any individual the size of the gap between capacity and performance. These factors are not well described in the literature, though self-esteem and self-concept have been explored (46).

We have illustrated how structural abnormalities in HCP impact on hand function, activity, and participation, and how personal and environmental factors influence this. There is a need for safe, cost-effective, evidence-based early interventions for infants and young children at risk of developing hemiplegia (47). Intervening early could break the vicious spiral of declining structure and function and improve long-term functional outcome. Optimizing hand and wrist posture could encourage the child to experiment more with using the affected limb, drive use-dependent plasticity toward optimal solutions, and reduce the risk of secondary musculoskeletal problems. Consideration should also be made regarding how to enhance visual and sensory awareness of the affected arm and hand and promote its use in play and other activities.

\section{ESTABLISHED MEDICAL AND SURGICAL INTERVENTIONS}

Outside the period of infancy, medical and surgical interventions are often considered; these include medications, targeted botulinum toxin treatment, and eventually surgical approaches, with ongoing therapy input and orthoses as appropriate. Botulinum toxin can reduce spasticity in targeted muscles and improve range of movement at a particular joint, facilitating splinting and therapy. This is a time-limited intervention: effects wear off and repeated administration is required. There is concern regarding the long-term effects of repeated injection, resulting in weakness and muscle atrophy in a group of children for whom muscle weakness is already a problematic feature (48). Botulinum toxin is both unlicensed and rarely used in practice for children age $<2$ years although a recent review of off-label botulinum toxin treatment identified three randomized controlled trials including children in this age range (49). Only one of these was relevant to upper limb function (50), this included children age 22-58 months. The study, while small, indicated a benefit from repeated botulinum toxin injections in the upper limb over and above that of occupational therapy alone, with respect to spasticity and parental perception of performance.

Surgical approaches include tendon transfer, muscle lengthening, and arthrodesis. These well-established procedures offer permanent and fixed solutions: in the case of arthrodesis, the overall range of movement is reduced. Treatment goals include achieving a more functional hand position and improving appearance or hygiene of the arm and hand. Robust studies of long-term outcome are sparse, but there is evidence of benefit (51-53). This includes perceived long-term benefit in terms of function and cosmesis from the patient perspective $(54,55)$. A full review of medical and surgical interventions is beyond the scope of this article. Below, we focus on therapy and splinting approaches and how these might apply to the infant population.

\section{THERAPY APPROACHES}

A number of strategies have been developed aiming to improve hand function in children with established HCP, with varying evidence of benefit (56). Current therapy approaches fundamentally comprise repeated practice of desired movements (sometimes including shaping, i.e., breaking down the goal into incremental steps in line with progress), with the child as an active participant. Most activities consider the principles of motor learning and neuroplasticity and are adapted to the age and cognitive ability of the child (57). Approaches may be play-based, problem-solving, or goal centered, for example, around specific activities of daily living (58). Some approaches such as the use of videogames/virtual reality, or robot-assisted therapy (sometimes in combination with virtual reality games), represent alternative modes of delivery of upper limb therapy rather than radically new principles per se (59). In older children with hemiplegia, there are now studies combining non-invasive brain stimulation with occupational therapy approaches.

Some therapies are delivered in intensive bursts (e.g., 60-90 h over a few weeks), which differs from more conventional therapy approaches $(57,60,61)$. Information regarding the optimal therapy "dose" is available for only a minority of interventions (62, 63). The practicalities of intensive therapy delivery must be considered in the light of other demands on the child, family, and therapist time as well as other resource pressures. Further information on the relative merits of intensive versus standard models of therapy will emerge in due course (64). A combined approach of infrequent intensive bursts of therapy, with lower-intensity "maintenance" therapy during interim periods, may prove optimal for enhancing and maintaining function.

Of key importance is the transfer of motor function gains from therapy to everyday life, without which there is little point in undertaking the therapy (65). This has been overtly assessed in the adult population in the context of constraint-induced movement therapy (CIMT - see below) (66). Incorporation of a "transfer package" with items such as a behavioral contract, home diary, task practice in the home setting, and ongoing telephone contact beyond the end of the therapy, greatly enhanced the improvements in the amount and quality of arm use compared with lab-based CIMT alone. There is indirect evidence from pediatric studies that similar principles apply (65).

\section{CHALLENGES OF EARLY THERAPY APPROACHES}

Developing upper limb therapies suitable for infants is a challenge for many obvious reasons. Infants have short attention spans and little or no understanding of the need for therapy or its aims. This makes it difficult to develop approaches which will be tolerated. Furthermore, evaluating the effects of therapy is tricky in a group with ongoing developmental changes in whom the outcome without intervention can vary. While there is a range of assessments of general motor function in infancy (currently validated to varying degrees), there is only one validated assessment 
of upper limb function - the Mini Assisting Hand Assessment, which measures function of the affected arm during structured bimanual play in infants 8-18 months (67). A further assessment, the Hand Assessment of Infants (HAI) for measuring unimanual and bimanual function in infants age 3-12 months, is in the late stages of development $(68,69)$. The grasping and reaching assessment of Brisbane ("GRAB") is being developed for infants from around 3 months (69) but this is also under development and not yet validated.

\section{CONSTRAINT-INDUCED MOVEMENT THERAPY AND BIMANUAL THERAPY}

Two therapy approaches, which have received much recent attention, are CIMT, in which the use of the less-affected hand and arm is restricted to encourage use of the more-affected upper limb, and intensive bimanual therapy (62). There is evidence of significant benefit from both of these approaches (56). Bimanual therapy may require a greater intervention duration (62). Guidelines for future research have been drawn up in relation to remaining unknowns regarding constraint therapy, including long-term benefits, effect of repeated programs, effect on bimanual performance, and activities of daily living, optimal type of restraint, type and duration of training, environment where the training is delivered and which patient characteristics influence outcome (70). The long-term effects on function of the less-affected limb must also be monitored (41).

Considering the infant population specifically, there has, to date, been no definitive evaluation of CIMT. However, infants have been included as part of trials studying a broader age group (8 months to 8 years) (71). Similarly, in a non-randomized trial, constraint improved the use of the hemiplegic hand in bimanual play compared with standard therapy approaches in children age 18 months to 4 years (72). The approaches and challenges of CIMT in young children with hemiplegia have been addressed by Taub et al. (73). These challenges are now being addressed in infants at risk of HCP: Eliasson et al. (68) will compare baby constraint-induced movement therapy (Baby-CIMT) with baby massage. Baby-CIMT is described as a modified CIMT protocol, the restraint being a mitt or glove. The intervention will be for 30 min daily for 6 weeks in infants age 3-8 months and will focus on grasping and exploring objects in an enriched environment with close attention to carers' and therapists' behavior toward the infant, toy selection and position of the infant (68). With respect to the need for development of movement control of the "unaffected" hand and for bimanual motor integration in infancy, caution is needed when considering CIMT in infants $(41,70)$; however, this protocol represents a much reduced intensity of intervention compared with classical CIMT, which would typically involve 60 or $90 \mathrm{~h}$ of therapy within a few weeks (63).

\section{ACTION OBSERVATION THERAPY}

"Action observation therapy" is a method aimed at stimulating the mirror neuron system - a frontoparietal network of neurons activated both during action observation and action performance. Mirror neurons were first identified by Rizzolatti in animal studies and were so named because they fired when the animal either performed a motor task or observed the same task being performed
(74). There is indirect evidence for the existence of a human mirror neuron system (75). Therapies incorporating repeated action observation and imitation showed promise in adults with stroke $(76,77)$.

Several small studies in children with HCP, generally adopting a bimanual approach in contrast to CIMT, also suggested that a benefit from action observation therapy (78-80). One larger study from our own group (ISRCTN65947097) in children with HCP age 3-10 years has also recently completed. There is to our knowledge only one study exploring the possible benefits of action observation therapy on upper limb function in infants at risk of developing HCP, as well as in healthy control infants (69). The intervention is targeted between the 9th and 13th weeks of life, during which parents will repeatedly demonstrate grasping actions on specific toys. This will be compared with presentations of the toys without demonstrating appropriate grasping actions. The effects on quantity and quality of reaching and grasping, as well as neurophysiological measures, will be assessed. Thus, at present, the role of action observation therapy, though intuitively appealing as a natural way of learning through watching and copying, remains to be established.

\section{HAND SPLINTS/ORTHOSES}

A splint has been broadly defined as "a brace, orthosis, cast, tape, or any external device applied to one or more joints" (81). Hand splints may be divided into "non-functional" splints aimed at preventing contracture by providing prolonged stretch, and "functional" splints aimed at improving motor task performance by supporting joints in biomechanically optimal positions (82). Although upper limb orthoses are frequently prescribed by therapists for children with hemiplegia, compliance overall is under $50 \%$ (83), with ineffectiveness and refusal to wear the orthosis being reasons for abandonment. Pain, discomfort, and reduction in function have been reported in the adult stroke literature (84). Additionally, by covering part of the palmar surface, thumb, and volar splints reduce palmar sensory feedback, which is vital for sensorimotor integration. All orthoses have the advantage that they can be worn for short periods of time rather than continuously, reducing the risk of disuse atrophy compared with approaches such as casting.

"Non-functional" orthoses offer a benign, temporary solution to correcting posture but are relatively inflexible. This reduces function and with excessive use may lead to muscle atrophy and compensatory overuse of other muscle groups (85). Therefore, non-functional orthoses are often used either at night or for short periods of time to achieve a particular goal, for example, increased muscle length. However, the evidence for this approach (86) or indeed for the benefit of non-functional orthoses (82) is limited. A recent systematic review found five randomized studies of nonfunctional upper limb splints (82), showing a small immediate benefit for hand function, which was not maintained. The review identified only one study of upper limb functional splinting, in which a Lycra splint from the wrist to the axilla had an additive benefit on goal attainment scores compared with goal-directed training alone (87).

An early description of a functional thumb splint was provided by Currie and Mendiola (88). The splint was assessed in just five 
children with HCP, aged 20-26 months. In each child this immediately improved thumb position, grasp type, and spontaneous bimanual exploration. More recently, Louwers et al. (89) studied the immediate effects of wearing a thumb and wrist brace on bimanual hand function in 25 children age $4-11$ years with HCP. Performance on the Assisting Hand Assessment (which measures the spontaneous use of the paretic hand to assist in tasks requiring bimanual function) (90) increased by an average of 3.2 raw scores simply by wearing the brace, and $52 \%$ of children improved by at least 4 points. No attempt to look at longer-term use, acceptability, or tolerability was made in either of these studies. Ten Berge et al. (91) studied the effects of a 2-month intervention with a neoprene thumb opponens splint on hand function in seven children age 2-7 years with hemiplegia. The splint was prescribed for at least $4 \mathrm{~h} /$ day. Compliance was good but goal attainment scores increased above baseline variability in only three patients.

Splints can be particularly challenging for use in young children, who may simply remove them. It is difficult to create wellfitting splints for very small hands and wrists given the nature and thickness of the materials used. Until a clearer evidence base emerges, decisions regarding splinting are fraught with uncertainty (82). In the light of the inadequate evidence base (82), Jackman et al. propose to undertake a study comparing the effects of functional hand splints alone, with task specific training alone, and with both approaches combined, in children age $4-15$ years (81).

\section{ELASTIC TAPING}

An approach increasingly being used for the upper limb in neurorehabilitation settings (92) but which, to date, has not been adequately evaluated, is the use of elastic taping. This differs from the more rigid forms of "athletic taping" originally developed for sports injuries. Hypoallergenic, waterproof versions of elastic tape are available, as are a range of colors, which can help in attracting the infant's visual attention (though natural, skin tone colors are sometimes more helpful for infants who tend to remove the tape). Specific manuals and training courses are in existence and various methods of application have been described, including a "thumb extension assist" approach for the thumb-in-palm deformity (93). With caution, parents could be trained in application by an appropriately qualified therapist. This is an important consideration because application needs to be repeated frequently (around twice a week) (94).

Many claims have been made regarding the potential benefits of functional elastic taping and these are counterbalanced by both a healthy skepticism and lack of clear evidence of efficacy (95). One of the more plausible stated mechanisms of action relevant to HCP is through enhancement of cutaneous sensory feedback via stretch applied to the skin during movement. For example, taping for the thumb-in-palm deformity includes a longitudinal strip under tension on the dorsum of the thumb, which could lead to increased firing of cutaneous afferents (mechanoreceptors) on the underlying skin during thumb flexion. This could lead to enhanced proprioceptive feedback $(96,97)$. In fact, skin strain patterns in the hand provide kinesthetic information taking precedence over that from muscle spindle and articular afferents in some situations (98); this kinesthetic role may have been underestimated in the past (99). Complex interactions at spinal cord level lead to integration of signals from the various proprioceptive afferents (100), which can then affect muscle spindle sensitivity through modulation of gamma motor neuron firing (101-103), and ultimately perhaps alter the balance of muscle activity to strengthen thumb extensors over time (104).

This provides the theory; however, the evidence in practice for kinesthetic benefits is lagging. While rigid, athletic tape can stabilize the ankle joint and improve proprioception $(105,106)$, studies of elastic taping have produced mixed results $(107,108)$. Additionally, studies in CP as well as in control populations at different ages are essential. This is because cutaneous reflexes show agedependent changes and are altered in CP. Short-latency cutaneous reflexes are known to be largest in the first year of life, and are also exaggerated in the presence of an upper motor neuron lesion (109). In infants and in CP, stimulation of the digits can produce short-latency excitation of both the forearm flexor and extensor muscles, leading to co-contraction. In the second year of life, longlatency cutaneous reflexes emerge, taking a supraspinal route to the cortex with the efferent volley through the corticospinal tract (109), thus, the long-latency cutaneous reflex is affected in patients with an upper motor neuron lesion. The degree to which patients with $\mathrm{CP}$ would demonstrate a kinesthetic advantage from taping is unclear.

Studies in children with CP have tended to focus on practical outcomes such as gross motor development. For example, Iosa et al. (110) studied the effect of taping at the ankle joint in eight children with unilateral spastic CP. Taping was undertaken for 6 months, and led to a greater increment in Gross Motor Function Measure (GMFM) scores (assessed with the tape off) than expected within that timescale, as well as improved gait. The mechanisms behind this were not explored. Interestingly, the only patient who did not improve had marked dyspraxia with sensory integration dysfunction. Similarly, Simsek et al. (111) applied tape for a 12week period to the paraspinal muscles, aiming to improve sitting posture in children with CP and GMFCS III-V. Fifteen children were randomized to the intervention and 15 were controls. Both groups also received physiotherapy. Sitting Assessment Scale scores (SAS) (112) did not differ at baseline but, were higher in the intervention group (with the tape removed) at 12 weeks. GMFM scores did not improve. Footer also found no benefit of paraspinal muscle taping on GMFM scores over a 12-week period (113).

Elastic taping has been used to improve upper extremity function in adults following stroke, with anecdotal benefit (92). It has also been used for the upper limb both proximally and distally in an acute rehabilitation setting in children with acquired brain injury (114). Children were tested with the Melbourne Assessment of Unilateral Upper Limb Function (MUUL) (115) first with the tape off then with tape applied according to perceived need. Significant improvements were seen both immediately and after 3 days. The range of different taping regimens and the sample heterogeneity pose some problems in interpretation. Mazzone et al. (116) also studied upper limb taping in 16 children with HCP, with a mean age of 3 years. They underwent a 17-month rehabilitation program with tape applied in the first and last 5 months and a 7-month washout period in the middle. Taping included the thumb and spiraled up the arm to the middle third of the humerus. The results were suggestive of improvement only in the 
taped periods, with the caveats that dropout rate was high (50\%) and the assessment used (MUUL) was not suitable for children aged below 5 years.

We are unaware of any other published literature on the effectiveness of elastic tape in improving hand and wrist position and function in infants with CP. As it is increasingly being used in practice, a formal evaluation would be timely to determine if children with thumb-in-palm deformity benefit from this intervention, and if so, how.

\section{CONCLUSION}

Early intervention for the upper limb in hemiplegia remains challenging, though progress is being made. We have touched earlier on the difficulties of outcome assessment in the youngest infants and children, which make evaluation of interventions very difficult. The diversity of the population under study, in terms of lesion type, differences in post-lesional reorganization, and the degree to which other factors such as vision, sensation, and cognitive ability impact on hand function, must also be considered. Studies tend to be small and are dominated by short-term outcomes (with the aim of avoiding confounders due to developmental trajectories), whereas the long-term outcome may be the more important consideration. Therapy approaches including CIMT and action observation therapy are now being actively explored in the youngest children and infants. Functional elastic taping has some potential merits in this age group but requires further investigation with properly controlled studies. These should include assessments which could shed light on the underlying mode of action.

\section{AUTHOR CONTRIBUTIONS}

Anna Purna Basu drafted the manuscript, to which Janice Pearse, Susan Kelly, Vicki Wisher, and Jill Kisler contributed substantially. All authors have seen, agree to, and are prepared to be accountable for the final version of the manuscript.

\section{ACKNOWLEDGMENTS}

Funder: British Academy of Childhood Disability (Paul Polani Research Award). Dr. Anna Purna Basu is funded by a Career Development Fellowship award from the National Institute for Health Research. The views expressed in this publication are those of the author and not necessarily those of the NHS, the National Institute for Health Research, or the Department of Health.

\section{REFERENCES}

1. Arner M, Eliasson AC, Nicklasson S, Sommerstein K, Hagglund G. Hand function in cerebral palsy. Report of 367 children in a population-based longitudinal health care program. J Hand Surg Am (2008) 33:1337-47. doi:10.1016/j.jhsa.2008.02.032

2. World Health Organization. International Classification of Functioning, Disability and Health: ICF. Geneva: WHO (2001).

3. Surveillance of Cerebral Palsy in Europe. Surveillance of cerebral palsy in Europe: a collaboration of cerebral palsy surveys and registers. Surveillance of Cerebral Palsy in Europe (SCPE). Dev Med Child Neurol (2000) 42:816-24. doi:10.1017/S0012162200001511

4. Lemon RN. Descending pathways in motor control. Annu Rev Neurosci (2008) 31:195-218. doi:10.1146/annurev.neuro.31.060407.125547

5. Feys H, Eyssen M, Jaspers E, Klingels K, Desloovere K, Molenaers G, et al. Relation between neuroradiological findings and upper limb function in hemiplegic cerebral palsy. Eur J Paediatr Neurol (2010) 14:169-77. doi:10.1016/j. ejpn.2009.01.004
6. Bleyenheuft Y, Gordon AM. Precision grip control, sensory impairments and their interactions in children with hemiplegic cerebral palsy: a systematic review. Res Dev Disabil (2013) 34:3014-28. doi:10.1016/j.ridd.2013.05.047

7. Auld ML, Boyd RN, Moseley GL, Ware RS, Johnston LM. Impact of tactile dysfunction on upper-limb motor performance in children with unilateral cerebral palsy. Arch Phys Med Rehabil (2012) 93:696-702. doi:10.1016/j.apmr. 2011.10.025

8. Auld ML, Boyd R, Moseley GL, Ware R, Johnston LM. Tactile function in children with unilateral cerebral palsy compared to typically developing children. Disabil Rehabil (2012) 34:1488-94. doi:10.3109/09638288.2011.650314

9. Wilke M, Staudt M, Juenger H, Grodd W, Braun C, Krageloh-Mann I. Somatosensory system in two types of motor reorganization in congenital hemiparesis: topography and function. Hum Brain Mapp (2009) 30:776-88. doi:10.1002/hbm.20545

10. Hoon AH Jr, Stashinko EE, Nagae LM, Lin DD, Keller J, Bastian A, et al. Sensory and motor deficits in children with cerebral palsy born preterm correlate with diffusion tensor imaging abnormalities in thalamocortical pathways. Dev Med Child Neurol (2009) 51:697-704. doi:10.1111/j.1469-8749.2009.03306.x

11. Rose S, Guzzetta A, Pannek K, Boyd R. MRI structural connectivity, disruption of primary sensorimotor pathways, and hand function in cerebral palsy. Brain Connect (2011) 1:309-16. doi:10.1089/brain.2011.0034

12. Gordon AM, Charles J, Steenbergen B. Fingertip force planning during grasp is disrupted by impaired sensorimotor integration in children with hemiplegic cerebral palsy. Pediatr Res (2006) 60:587-91. doi:10.1203/01.pdr.0000242370. 41469.74

13. Guzzetta A, Fazzi B, Mercuri E, Bertuccelli B, Canapicchi R, van Hof-van Duin $\mathrm{J}$, et al. Visual function in children with hemiplegia in the first years of life. Dev Med Child Neurol (2001) 43:321-9. doi:10.1111/j.1469-8749.2001.tb00212.x

14. Steenbergen B, Jongbloed-Pereboom M, Spruijt S, Gordon AM. Impaired motor planning and motor imagery in children with unilateral spastic cerebral palsy: challenges for the future of pediatric rehabilitation. Dev Med Child Neurol (2013) 55(Suppl 4):43-6. doi:10.1111/dmcn.12306

15. Kirkpatrick EV, Pearse JE, Eyre JA, Basu AP. Motor planning ability is not related to lesion side or functional manual ability in children with hemiplegic cerebral palsy. Exp Brain Res (2013) 231:239-47. doi:10.1007/s00221-013-3687-x

16. Bodimeade HL, Whittingham K, Lloyd O, Boyd RN. Executive function in children and adolescents with unilateral cerebral palsy. Dev Med Child Neurol (2013) 55:926-33. doi:10.1111/dmcn.12195

17. Neville B, Goodman R. Congenital Hemiplegia. London: MacKeith Press (2000).

18. Ertelt D, Hemmelmann C, Dettmers C, Ziegler A, Binkofski F. Observation and execution of upper-limb movements as a tool for rehabilitation of motor deficits in paretic stroke patients: protocol of a randomized clinical trial. BMC Neurol (2012) 12:42. doi:10.1186/1471-2377-12-42

19. Eyre J. Development and plasticity of the corticospinal system in man. Neural Plast (2003) 10:93-106. doi:10.1155/NP.2003.93

20. Eyre J, Taylor J, Villagra F, Smith M, Miller S. Evidence of activity-dependent withdrawal of corticospinal projections during human development. Neurology (2001) 57:1543-54. doi:10.1212/WNL.57.9.1543

21. Eyre JA, Smith M, Dabydeen L, Clowry GJ, Petacchi E, Battini R, et al. Is hemiplegic cerebral palsy equivalent to amblyopia of the corticospinal system? Ann Neurol (2007) 62:493-503. doi:10.1002/ana.21108

22. Staudt M, Gerloff C, Grodd W, Holthausen H, Niemann G, Krageloh-Mann I. Reorganization in congenital hemiparesis acquired at different gestational ages. Ann Neurol (2004) 56:854-63. doi:10.1002/ana.20297

23. Basu A, Graziadio S, Smith M, Clowry GJ, Cioni G, Eyre JA. Developmental plasticity connects visual cortex to motoneurons after stroke. Ann Neurol (2010) 67:132-6. doi:10.1002/ana.21827

24. Clowry GJ, Davies BM, Upile NS, Gibson CL, Bradley PM. Spinal cord plasticity in response to unilateral inhibition of the rat motor cortex during development: changes to gene expression, muscle afferents and the ipsilateral corticospinal projection. Eur J Neurosci (2004) 20:2555-66. doi:10.1111/j.1460-9568.2004. 03713.x

25. Clowry GJ. The dependence of spinal cord development on corticospinal input and its significance in understanding and treating spastic cerebral palsy. Neurosci Biobehav Rev (2007) 31:1114-24. doi:10.1016/j.neubiorev.2007.04.007

26. Chakrabarty S, Shulman B, Martin JH. Activity-dependent codevelopment of the corticospinal system and target interneurons in the cervical spinal cord. J Neurosci (2009) 29:8816-27. doi:10.1523/JNEUROSCI.0735-09.2009 
27. Chakrabarty S, Friel KM, Martin JH. Activity-dependent plasticity improves M1 motor representation and corticospinal tract connectivity. J Neurophysiol (2009) 101:1283-93. doi:10.1152/jn.91026.2008

28. Friel K, Chakrabarty S, Kuo HC, Martin J. Using motor behavior during an early critical period to restore skilled limb movement after damage to the corticospinal system during development. J Neurosci (2012) 32:9265-76. doi:10.1523/JNEUROSCI.1198-12.2012

29. Guzzetta A, Mercuri E, Rapisardi G, Ferrari F, Roversi MF, Cowan F, et al. General movements detect early signs of hemiplegia in term infants with neonatal cerebral infarction. Neuropediatrics (2003) 34:61-6. doi:10.1055/s2003-39597

30. Jaffe M, Tal Y, Dabbah H, Ganelis L, Cohen A, Even L, et al. Infants with a thumb-in-fist posture. Pediatrics (2000) 105:E41. doi:10.1542/peds.105.3.e41

31. Park ES, Sim EG, Rha DW. Effect of upper limb deformities on gross motor and upper limb functions in children with spastic cerebral palsy. Res Dev Disabil (2011) 32:2389-97. doi:10.1016/j.ridd.2011.07.021

32. Koman LA, Smith BP, Shilt JS. Cerebral palsy. Lancet (2004) 363:1619-31. doi:10.1016/S0140-6736(04)16207-7

33. Carlson MG, Gallagher K, Spirtos M. Surgical treatment of swan-neck deformity in hemiplegic cerebral palsy. J Hand Surg Am (2007) 32:1418-22. doi:10.1016/j.jhsa.2007.07.002

34. House JH, Gwathmey FW, Fidler MO. A dynamic approach to the thumb-in palm deformity in cerebral palsy. J Bone Joint Surg Am (1981) 63:216-25.

35. Foley J. The stiffness of spastic muscle. J Neurol Neurosurg Psychiatry (1961) 24:125-31. doi:10.1136/jnnp.24.2.125

36. Lorentzen J, Grey MJ, Crone C, Mazevet D, Biering-Sorensen F, Nielsen JB. Distinguishing active from passive components of ankle plantar flexor stiffness in stroke, spinal cord injury and multiple sclerosis. Clin Neurophysiol (2010) 121:1939-51. doi:10.1016/j.clinph.2010.02.167

37. Damiano DL, Dodd K, Taylor NF. Should we be testing and training muscle strength in cerebral palsy? Dev Med Child Neurol (2002) 44:68-72. doi:10.1017/S0012162201001682

38. Autti-Ramo I, Larsen A, Taimo A, von Wendt L. Management of the upper limb with botulinum toxin type A in children with spastic type cerebral palsy and acquired brain injury: clinical implications. Eur J Neurol (2001) 8(Suppl 5):136-44. doi:10.1046/j.1468-1331.2001.00046.x

39. Sakzewski L, Ziviani J, Boyd R. The relationship between unimanual capacity and bimanual performance in children with congenital hemiplegia. Dev Med Child Neurol (2010) 52:811-6. doi:10.1111/j.1469-8749.2009.03588.x

40. Steenbergen B, Meulenbroek RG. Deviations in upper-limb function of the lessaffected side in congenital hemiparesis. Neuropsychologia (2006) 44:2296-307. doi:10.1016/j.neuropsychologia.2006.05.016

41. Basu A, Eyre J. A plea for consideration of the less affected hand in therapeutic approaches to hemiplegia. Dev Med Child Neurol (2012) 54:380 doi:10.1111/j.1469-8749.2012.04242.x

42. Fedrizzi E, Pagliano E, Andreucci E, Oleari G. Hand function in children with hemiplegic cerebral palsy: prospective follow-up and functional outcome in adolescence. Dev Med Child Neurol (2003) 45:85-91. doi:10.1111/j.1469-8749. 2003.tb00910.x

43. Zielinski IM, Jongsma ML, Baas CM, Aarts PB, Steenbergen B. Unravelling developmental disregard in children with unilateral cerebral palsy by measuring event-related potentials during a simple and complex task. BMC Neurol (2014) 14:6. doi:10.1186/1471-2377-14-6

44. Van Zelst BR, Miller MD, Russo R, Murchland S, Crotty M. Activities of daily living in children with hemiplegic cerebral palsy: a cross-sectional evaluation using the assessment of motor and process skills. Dev Med Child Neurol (2006) 48:723-7. doi:10.1017/S0012162206001551

45. Skold A, Josephsson S, Eliasson AC. Performing bimanual activities: the experiences of young persons with hemiplegic cerebral palsy. Am J Occup Ther (2004) 58:416-25. doi:10.5014/ajot.58.4.416

46. Russo RN, Goodwin EJ, Miller MD, Haan EA, Connell TM, Crotty M. Selfesteem, self-concept, and quality of life in children with hemiplegic cerebral palsy. J Pediatr (2008) 153:473-7. doi:10.1016/j.jpeds.2008.05.040

47. Basu AP. Early intervention after perinatal stroke: opportunities and challenges. Dev Med Child Neurol (2014) 56:516-21. doi:10.1111/dmcn.12407

48. Edgar TS. Clinical utility of botulinum toxin in the treatment of cerebral palsy: comprehensive review. J Child Neurol (2001) 16:37-46. doi:10.1177/ 088307380101600107
49. Druschel C, Althuizes HC, Funk JF, Placzek R. Off label use of botulinum toxin in children under two years of age: a systematic review. Toxins (Basel) (2013) 5:60-72. doi:10.3390/toxins5010060

50. Olesch CA, Greaves S, Imms C, Reid SM, Graham HK. Repeat botulinum toxin-A injections in the upper limb of children with hemiplegia: a randomized controlled trial. Dev Med Child Neurol (2010) 52:79-86. doi:10.1111/j. 1469-8749.2009.03387.x

51. Smitherman JA, Davids JR, Tanner S, Hardin JW, Wagner LV, Peace LC, et al. Functional outcomes following single-event multilevel surgery of the upper extremity for children with hemiplegic cerebral palsy. J Bone Joint Surg Am (2011) 93:655-61. doi:10.2106/JBJS.J.00295

52. Eliasson AC, Ekholm C, Carlstedt T. Hand function in children with cerebral palsy after upper-limb tendon transfer and muscle release. Dev Med Child Neurol (1998) 40:612-21. doi:10.1111/j.1469-8749.1998.tb15427.x

53. Skold A, Ekholm C, Eliasson AC. Improved hand function remains after upperlimb tendon transfer and muscle release in children with hemiplegia. Dev Med Child Neurol (1999) 41:284-5. doi:10.1017/S0012162299210602

54. Skold A, Josephsson S, Fitinghoff H, Eliasson AC. Experiences of use of the cerebral palsy hemiplegic hand in young persons treated with upper extremity surgery. J Hand Ther (2007) 20:262-72. doi:10.1197/j.jht.2007.04.003

55. Johnstone BR, Richardson PW, Coombs CJ, Duncan JA. Functional and cosmetic outcome of surgery for cerebral palsy in the upper limb. Hand Clin (2003) 19:679-86. doi:10.1016/S0749-0712(03)00031-3

56. Sakzewski L, Ziviani J, Boyd RN. Efficacy of upper limb therapies for unilateral cerebral palsy: a meta-analysis. Pediatrics (2014) 133:e175-204. doi:10.1542/ peds.2013-0675

57. Novak I. A magical moment in research translation: strategies for providing high intensity bimanual therapy. Dev Med Child Neurol (2013) 55:491. doi: $10.1111 / \mathrm{dmcn} .12082$

58. Mastos M, Miller K, Eliasson AC, Imms C. Goal-directed training: linking theories of treatment to clinical practice for improved functional activities in daily life. Clin Rehabil (2007) 21:47-55. doi:10.1177/0269215506073494

59. Chen YP, Howard AM. Effects of robotic therapy on upper-extremity function in children with cerebral palsy: a systematic review. Dev Neurorehabil (2014) doi:10.3109/17518423.2014.899648

60. Novak I, McIntyre S, Morgan C, Campbell L, Dark L, Morton N, et al. A systematic review of interventions for children with cerebral palsy: state of the evidence. Dev Med Child Neurol (2013) 55:885-910. doi:10.1111/dmcn.12246

61. Gordon AM, Hung YC, Brandao M, Ferre CL, Kuo HC, Friel K, et al. Bimanual training and constraint-induced movement therapy in children with hemiplegic cerebral palsy: a randomized trial. Neurorehabil Neural Repair (2011) 25:692-702. doi:10.1177/1545968311402508

62. Gordon AM. To constrain or not to constrain, and other stories of intensive upper extremity training for children with unilateral cerebral palsy. Dev Med Child Neurol (2011) 53(Suppl 4):56-61. doi:10.1111/j.1469-8749.2011.04066.x

63. Kolobe TH, Christy JB, Gannotti ME, Heathcock JC, Damiano DL, Taub E, et al. Research summit III proceedings on dosing in children with an injured brain or cerebral palsy: executive summary. Phys Ther (2014) 94:907-20. doi:10.2522/ptj.20130024

64. Boyd RN, Ziviani J, Sakzewski L, Miller L, Bowden J, Cunnington R, et al. COMBIT: protocol of a randomised comparison trial of COMbined modified constraint induced movement therapy and bimanual intensive training with distributed model of standard upper limb rehabilitation in children with congenital hemiplegia. BMC Neurol (2013) 13:68. doi:10.1186/1471-2377-13-68

65. Taub E, Uswatte G. Importance for CP rehabilitation of transfer of motor improvement to everyday life. Pediatrics (2014) 133:e215-7. doi:10.1542/peds. 2013-3411

66. Taub E, Uswatte G, Mark VW, Morris DM, Barman J, Bowman MH, et al. Method for enhancing real-world use of a more affected arm in chronic stroke: transfer package of constraint-induced movement therapy. Stroke (2013) 44:1383-8. doi:10.1161/STROKEAHA.111.000559

67. Greaves S, Imms C, Dodd K, Krumlinde-Sundholm L. Development of the mini-assisting hand assessment: evidence for content and internal scale validity. Dev Med Child Neurol (2013) 55:1030-7. doi:10.1111/dmcn.12212

68. Eliasson AC, Sjostrand L, Ek L, Krumlinde-Sundholm L, Tedroff K. Efficacy of baby-CIMT: study protocol for a randomised controlled trial on infants below age 12 months, with clinical signs of unilateral CP. BMC Pediatr (2014) 14:141. doi:10.1186/1471-2431-14-141 
69. Guzzetta A, Boyd RN, Perez M, Ziviani J, Burzi V, Slaughter V, et al. UP-BEAT (upper limb baby early action-observation training): protocol of two parallel randomised controlled trials of action-observation training for typically developing infants and infants with asymmetric brain lesions. BMJ Open (2013) 3:e002512. doi:10.1136/bmjopen-2012-002512

70. Eliasson AC, Krumlinde-Sundholm L, Gordon AM, Feys H, Klingels K, Aarts $\mathrm{PB}$, et al. Guidelines for future research in constraint-induced movement therapy for children with unilateral cerebral palsy: an expert consensus. Dev Med Child Neurol (2014) 56:125-37. doi:10.1111/dmcn.12273

71. Taub E, Ramey SL, DeLuca S, Echols K. Efficacy of constraint-induced movement therapy for children with cerebral palsy with asymmetric motor impairment. Pediatrics (2004) 113:305-12. doi:10.1542/peds.113.2.305

72. Eliasson AC, Krumlinde-sundholm L, Shaw K, Wang C. Effects of constraintinduced movement therapy in young children with hemiplegic cerebral palsy: an adapted model. Dev Med Child Neurol (2005) 47:266-75. doi:10.1111/j. 1469-8749.2005.tb01132.x

73. Taub E, Griffin A, Nick J, Gammons K, Uswatte G, Law CR. Pediatric CI therapy for stroke-induced hemiparesis in young children. Dev Neurorehabil (2007) 10:3-18. doi:10.1080/13638490601151836

74. Rizzolatti G, Craighero L. The mirror-neuron system. Annu Rev Neurosci (2004) 27:169-92. doi:10.1146/annurev.neuro.27.070203.144230

75. Kilner JM, Lemon RN. What we know currently about mirror neurons. Curr Biol (2013) 23:R1057-62. doi:10.1016/j.cub.2013.10.051

76. Ertelt D, Small S, Solodkin A, Dettmers C, McNamara A, Binkofski F, et al. Action observation has a positive impact on rehabilitation of motor deficits after stroke. Neuroimage (2007) 36(Suppl 2):T164-73. doi:10.1016/j. neuroimage.2007.03.043

77. Franceschini M, Ceravolo MG, Agosti M, Cavallini P, Bonassi S, Dall'Armi $\mathrm{V}$, et al. Clinical relevance of action observation in upper-limb stroke rehabilitation: a possible role in recovery of functional dexterity. A randomized clinical trial. Neurorehabil Neural Repair (2012) 26:456-62. doi:10.1177/ 1545968311427406

78. Sgandurra G, Cecchi F, Serio SM, Del Maestro M, Laschi C, Dario P, et al. Longitudinal study of unimanual actions and grasping forces during infancy. Infant Behav Dev (2012) 35:205-14. doi:10.1016/j.infbeh.2012.01.003

79. Buccino G, Arisi D, Gough P, Aprile D, Ferri C, Serotti L, et al. Improving upper limb motor functions through action observation treatment: a pilot study in children with cerebral palsy. Dev Med Child Neurol (2012) 54:822-8. doi:10.1111/j.1469-8749.2012.04334.x

80. Kim JY, Kim JM, Ko EY. The effect of the action observation physical training on the upper extremity function in children with cerebral palsy. J Exerc Rehabil (2014) 10:176-83. doi:10.12965/jer.140114

81. Jackman M, Novak I, Lannin N. Effectiveness of functional hand splinting and the cognitive orientation to occupational performance (CO-OP) approach in children with cerebral palsy and brain injury: two randomised controlled trial protocols. BMC Neurol (2014) 14:144. doi:10.1186/1471-2377-14-144

82. Jackman M, Novak I, Lannin N. Effectiveness of hand splints in children with cerebral palsy: a systematic review with meta-analysis. Dev Med Child Neurol (2014) 56(2):138-47. doi:10.1111/dmcn.12205

83. Russo RN, Atkins R, Haan E, Crotty M. Upper limb orthoses and assistive technology utilization in children with hemiplegic cerebral palsy recruited from a population register. Dev Neurorehabil (2009) 12:92-9. doi:10.1080/ 17518420902783223

84. Andringa AS, Van de Port IG, Meijer JW. Tolerance and effectiveness of a new dynamic hand-wrist orthosis in chronic stroke patients. NeuroRehabilitation (2013) 33:225-31. doi:10.3233/NRE- 130949

85. Burtner PA, Poole JL, Torres T, Medora AM, Abeyta R, Keene J, et al. Effect of wrist hand splints on grip, pinch, manual dexterity, and muscle activation in children with spastic hemiplegia: a preliminary study. J Hand Ther (2008) 21:36-42. doi:10.1197/j.jht.2007.08.018

86. Lannin NA, Ada L. Neurorehabilitation splinting: theory and principles of clinical use. NeuroRehabilitation (2011) 28:21-8. doi:10.3233/NRE-2011-0628

87. Elliott CM, Reid SL, Alderson JA, Elliott BC. Lycra arm splints in conjunction with goal-directed training can improve movement in children with cerebral palsy. NeuroRehabilitation (2011) 28:47-54. doi:10.3233/NRE2011-0631

88. Currie DM, Mendiola A. Cortical thumb orthosis for children with spastic hemiplegic cerebral palsy. Arch Phys Med Rehabil (1987) 68:214-6.
89. Louwers A, Meester-Delver A, Folmer K, Nollet F, Beelen A. Immediate effect of a wrist and thumb brace on bimanual activities in children with hemiplegic cerebral palsy. Dev Med Child Neurol (2011) 53:321-6. doi:10.1111/j.14698749.2010.03849.x

90. Krumlinde-Sundholm L, Holmefur M, Kottorp A, Eliasson AC. The assisting hand assessment: current evidence of validity, reliability, and responsiveness to change. Dev Med Child Neurol (2007) 49:259-64. doi:10.1111/j.1469-8749. 2007.00259.x

91. Ten Berge SR, Boonstra AM, Dijkstra PU, Hadders-Algra M, Haga N, Maathuis CG. A systematic evaluation of the effect of thumb opponens splints on hand function in children with unilateral spastic cerebral palsy. Clin Rehabil (2012) 26:362-71. doi:10.1177/0269215511411936

92. Jaraczewska E, Long C. Kinesio taping in stroke: improving functional use of the upper extremity in hemiplegia. Top Stroke Rehabil (2006) 13:31-42. doi:10.1310/33KA-XYE3-QWJB-WGT6

93. Kase K, Martin P, Yasukawa A. Kinesio Taping ${ }^{\circledR}$ in Pediatrics. Tokyo: Ken Ikai Co Ltd (2006).

94. Grandi A, Mazzola M, Angelini L, Chiappedi M. Listening to the patient as a possible route to cost-effective rehabilitation: a case report. J Med Case Rep (2012) 6:19. doi:10.1186/1752-1947-6-19

95. Morris D, Jones D, Ryan H, Ryan CG. The clinical effects of Kinesio(R) Tex taping: a systematic review. Physiother Theory Pract (2013) 29:259-70. doi:10.3109/09593985.2012.731675

96. Gandevia SC, Hall LA, McCloskey DI, Potter EK. Proprioceptive sensation at the terminal joint of the middle finger. J Physiol (1983) 335:507-17.

97. Edin BB. Quantitative analyses of dynamic strain sensitivity in human skin mechanoreceptors. J Neurophysiol (2004) 92:3233-43. doi:10.1152/jn. 00628.2004

98. Edin BB, Johansson N. Skin strain patterns provide kinaesthetic information to the human central nervous system. J Physiol (1995) 487(Pt 1):243-51.

99. Burke D, Gandevia SC, Macefield G. Responses to passive movement of receptors in joint, skin and muscle of the human hand. J Physiol (1988) 402:347-61.

100. Pierrot-Deseilligny E, Bergego C, Katz R, Morin C. Cutaneous depression of Ib reflex pathways to motoneurones in man. Exp Brain Res (1981) 42: 351-61.

101. Riemann BL, Lephart SM. The sensorimotor system, part I: the physiologic basis of functional joint stability. J Athl Train (2002) 37:71-9.

102. Riemann BL, Lephart SM. The sensorimotor system, part II: the role of proprioception in motor control and functional joint stability. J Athl Train (2002) 37:80-4.

103. Johansson H, Sojka P. Actions on gamma-motoneurones elicited by electrical stimulation of cutaneous afferent fibres in the hind limb of the cat. J Physiol (1985) 366:343-63.

104. Gomez-Soriano J, Abian-Vicen J, Aparicio-Garcia C, Ruiz-Lazaro P, SimonMartinez C, Bravo-Esteban E, et al. The effects of Kinesio taping on muscle tone in healthy subjects: a double-blind, placebo-controlled crossover trial. Man Ther (2014) 19:131-6. doi:10.1016/j.math.2014.06.004

105. Lohrer H, Alt W, Gollhofer A. Neuromuscular properties and functional aspects of taped ankles. Am J Sports Med (1999) 27:69-75.

106. Robbins S, Waked E, Rappel R. Ankle taping improves proprioception before and after exercise in young men. Br J Sports Med (1995) 29:242-7. doi:10.1136/ bjsm.29.4.242

107. Halseth T, McChesney JW, Debeliso M, Vaughn R, Lien J. The effects of Kinesio taping on proprioception at the ankle. J Sports Sci Med (2004) 3:1-7.

108. Chang HY, Chou KY, Lin JJ, Lin CF, Wang CH. Immediate effect of forearm Kinesio taping on maximal grip strength and force sense in healthy collegiate athletes. Phys Ther Sport (2010) 11:122-7. doi:10.1016/j.ptsp.2010. 06.007

109. Issler H, Stephens JA. The maturation of cutaneous reflexes studied in the upper limb in man. J Physiol (1983) 335:643-54.

110. Iosa M, Morelli D, Nanni MV, Veredice C, Marro T, Medici A, et al. Functional taping: a promising technique for children with cerebral palsy. Dev Med Child Neurol (2010) 52:587-9. doi:10.1111/j.1469-8749.2009.03539.x

111. Simsek TT, Turkucuoglu B, Cokal N, Ustunbas G, Simsek IE. The effects of Kinesio(R) taping on sitting posture, functional independence and gross motor function in children with cerebral palsy. Disabil Rehabil (2011) 33:2058-63. doi:10.3109/09638288.2011.560331 
112. Myhr U, von Wendt L. Improvement of functional sitting position for children with cerebral palsy. Dev Med Child Neurol (1991) 33:246-56. doi:10.1111/j. 1469-8749.1991.tb05114.x

113. Footer CB. The effects of therapeutic taping on gross motor function in children with cerebral palsy. Pediatr Phys Ther (2006) 18:245-52. doi:10.1097/01. pep.0000233696.33675.37

114. Yasukawa A, Patel P, Sisung C. Pilot study: investigating the effects of Kinesio taping in an acute pediatric rehabilitation setting. Am J Occup Ther (2006) 60:104-10. doi:10.5014/ajot.60.1.104

115. Randall MJ, Johnson LM, Reddihough DS. The Melbourne Assessment of Unilateral Upper Limb Function: Test Administration Manual. Melbourne, VIC: Royal Children's Hospital (1999).

116. Mazzone S, Serafini A, Iosa M, Aliberti MN, Gobbetti T, Paolucci S, et al. Functional taping applied to upper limb of children with hemiplegic cerebral palsy: a pilot study. Neuropediatrics (2011) 42:249-53. doi:10.1055/s-00311295478

Conflict of Interest Statement: The Guest Associate Editor Gavin John Clowry declares that, despite having previously collaborated with Anna Purna Basu and Janice Pearse and being affiliated to the same institution as author Jill Kisler, the review process was handled objectively and no conflict of interest exists. The authors received funding from the British Academy of Childhood Disability/Royal College of Pediatrics and Child Health (Paul Polani award) to undertake a pilot study of functional taping to improve thumb and wrist posture and function in children with cerebral palsy.

Received: 19 August 2014; accepted: 09 December 2014; published online: 06 January 2015.

Citation: Basu AP, Pearse J, Kelly S, Wisher V and Kisler J (2015) Early intervention to improve hand function in hemiplegic cerebral palsy. Front. Neurol. 5:281. doi: 10.3389/fneur.2014.00281

This article was submitted to Neuropediatrics, a section of the journal Frontiers in Neurology.

Copyright (c) 2015 Basu, Pearse, Kelly, Wisher and Kisler. This is an open-access article distributed under the terms of the Creative Commons Attribution License (CC BY). The use, distribution or reproduction in other forums is permitted, provided the original author(s) or licensor are credited and that the original publication in this journal is cited, in accordance with accepted academic practice. No use, distribution or reproduction is permitted which does not comply with these terms. 